Haro, G., and Rivera Terrazas, L. (1954).-Ton. y Tac., Bol. No. 10: 3-15.

Herbia, G. H. (1962).-Ap. J. 135 : 736-47.

Johnson, H. L., and Mrtchels, R. I. (1958).-Ap. J. 128 : 31-40.

Joy, A. H. (1949).-Ap. J. 110 : 424-37.

JoY, A. H. (1960)._-"Stars and Stellar Systems." (General Ed. G. P. Kuiper.) Vol. VI. pp. 653-75. (Univ. Chicago Press.)

Oskanjan, V. (1957a).—“Non-stable Stars." pp. 17-27. [Byurakan 1956.] (Acad. Sci. Armenian SSR: Erevan.)

OsKanjaN, V. (1957b). -Personal communication.

Petit, M. (1958)._“Colloqui d'Astrofisica di Asiago (1956-1958).” Asiago Contr. No. 95: $29-44$.

Rosino, L. (1956).-Asiago Contr. No. 69.

Rosino, L., and Cian, A. (1962).-Asiago Contr. No. 125.

Rosino, L., Grubissich, C., and MafFer, P. (1957).-Asiago Contr. No. 82.

\title{
Discussion
}

Buscombe: Has any search been made for flash stars in II Scorpii, where Struve had previously found T-Tauri stars? Pleiades.

Haro: No, only in the Orion nebula, in NGC 2264, the Taurus dark clouds, and in the

Haro: I would like to state that the Tonantzintla Observatory and Sagamore Hill Radio Observatory have collaborated in observing flash stars in the Orion nebula. The purpose of the radio and optical observations over the Orion and Pleiades clusters, which will be continued at the end of the year, is to demonstrate that a radio outburst in a flash star can be detected as far as the distance of the Pleiades and the Orion nebula.

Slee: Simultaneous radio and optical observations made on the flare star V371 Orionis in November 1962 resulted in the detection of radio bursts at $75 \mathrm{~cm}$ and 15 metres. The results are at present provisional because the detailed light curve of the event, obtained with the BakerNunn Schmidt camera operated by the Smithsonian Institution at Woomera, is not yet available.

\section{GALACTIC EVOLUTION AND THE TWO-COLOUR DIAGRAM}

\author{
R. H. Stoy \\ Royal Observatory, Cape Town
}

Information about the past history of the Galaxy may be obtained from a two-colour diagram, since the present distribution of stars in such a diagram depends on the past rate of star formation and the past metal abundances in the interstellar medium. As an illustration of this, I would like to discuss three diagrams that were recently prepared by Dr. M. E. Dixon from Cape photometric data (Dixon $1963 a, b)$.

In the first diagram $(U-B)_{\mathrm{C}}$ is plotted against $(B-V)$ for all single stars of known MK type belonging to luminosity classes V and VI and lying within 100 pc of the Sun. Class VI is taken as referring to subdwarfs, particularly those with sufficiently extreme metal deficiencies to be detected as such by their spectra. The particular feature in this diagram to which $I$ wish to draw attention is the way in which the left-hand boundary of the domain in which the Class VI stars fall coincides with the "blanketing line" defined by

$$
(B-V)_{\text {Hyades }}=0 \cdot 60 \text {. }
$$

